\title{
La bioética en la empresa: el caso particular de la Responsabilidad Social Corporativa
}

\author{
María Peana Chivite, Sara Gallardo \\ Universidad Católica «Sta. Teresa de Jesús» de Ávila \\ mpeana.chivite@ucavila.es, sara.gallardo@ucavila.es
}

Resumen: Dada la importancia de la bioética para tratar de evitar los abusos cometidos en el pasado o los nuevos abusos que pueden darse por los avances de la ciencia, este trabajo de investigación busca fomentar la difusión y divulgación de esta disciplina, particularmente en las empresas que tienen una relación más directa con la investigación o promoción de la vida del ser bumano. Para ello, se propone otra realidad paralela que está cobrando en la actualidad una gran importancia y una adopción creciente por parte de las empresas: la Responsabilidad Social Corporativa (RSC).

Palabras clave: responsabilidad social de la empresa, ética, industria farmacéutica, biotecnología.

Bioethics in Business: the case of Corporate Social Responsibility

Abstract: Given the importance of bioethics in preventing the repetition of abuses committed in the past and new abuses that may arise through scientific advances, this research article aims to encourage the dissemination of bioetbics, particularly in companies that have a more direct relation with research into and the promotion of buman life. To do so, the article proposes the adoption of a parallel approach that has become highly important and is increasingly being adopted by many companies: Corporate Social Responsibility (RSC).

Keywords: company social responsibility, etbics, pharmaceutical industry, biotechnology. 


\section{Introducción}

La bioética es en la actualidad un tema de interés creciente, ya que la vida humana está cada vez más envuelta en nuevas situaciones, donde posibilidades antes inexistentes en medicina y experimentación - positivas y negativas - nos confrontan con cuestiones que antes no podíamos ni plantearnos en relación con el comienzo y el fin de la propia vida o con la enfermedad. Como señala Sgreccia (2007), esta disciplina tiene unas raíces remotas y otras recientes, por ejemplo, los abusos cometidos por los médicos nazis en los campos de concentración, algunas experimentaciones realizadas en EE. UU. sin el consentimiento de las personas, como, en los años 30 y 40 en Tuskegee, Alabama, un estudio comparativo de fármacos sobre población de color o, en 1963 en el Jewish Chronic Disease Hospital de Brooklyn, la inyección de células tumorales a pacientes ancianos, entre otros. La necesidad de que se contemplen unos principios morales en el ámbito de la vida humana se hizo patente y dio lugar a distintos códigos bioéticos (Código de Núremberg, 1947, o Declaración de Helsinki, 1964 y 2000) y a la creación de institutos, ${ }^{1}$ comités de bioética o programas universitarios.

Dada la relevancia de la bioética para ayudar a que las ciencias fomenten y no destruyan la integridad y la dignidad de la vida de las personas, ${ }^{2}$ este trabajo pretende impulsar la difusión y divulgación de esta disciplina en algunas de las empresas que tienen una relación más directa con la investigación o promoción de la vida del ser humano.

Para ello, se propone otra realidad paralela que está adquiriendo hoy un gran valor y una adopción creciente por parte de las empresas: la Responsabilidad Social Corporativa (RSC); así lo muestran trabajos como los de Friedman (1970), McGuire et alii (1988), Carroll (1999), McWilliams et alii (2006), Porter y Kramer (2006), RACEF (2007), Alberich (2014), entre muchos otros. Según esta concepción, la empresa va más allá de la búsqueda de una rentabilidad económica y considera que tiene una responsabilidad con la sociedad con la que se relaciona y de la que obtiene beneficios. Esta responsabilidad la lleva a considerar el deber de contribuir a la mejora no solo del entorno, sino también de la misma sociedad. De este modo, la empresa y la sociedad en su conjunto mejoran, crecen y se desarrollan.

Lo que se persigue en esta investigación es, por un lado, hacer un análisis de la realidad de la RSC para comprobar si la bioética tiene cabida en esta concepción.

1 1968: Fundación del Hastings Center (Hastings, Hudson) por obra de D. Callahan y W. Gaylin.

1969: Fundación del Kennedy Institute of Ethics en la Georgetown University (Washington DC) por obra de A. Hellegers (W.T. Reich, R. Veatch, R. McCormick) (Sgreccia, 2007).

2 La Ley 14/2007, de 3 de julio, de Investigación Biomédica, es otro ejemplo de dicha actualidad y relevancia. 
Por otro lado, comprobar si las empresas que ya aplican RSC son más sensibles a la adopción o consideración de factores bioéticos en su actividad.

De modo que, si se muestra que la bioética debe formar parte de la responsabilidad de las empresas ${ }^{3}$ y que aquellas empresas que adoptan RSC, a su vez, tienden a incluir consideraciones bioéticas en su actividad, favorecer la divulgación de la RSC ayudaría a la expansión de la bioética, y más aún si se incluye dicho apartado explícitamente en esta realidad.

También sería interesante comprobar si las empresas más relacionadas con la investigación o promoción de la vida adoptan RSC, de modo complementario y en relación con dicho objetivo. Para ello, se tomarán las empresas farmacéuticasbiotecnológicas españolas que cotizan en bolsa.

\section{Marco teórico y conceptos básicos}

\subsection{Bioética y teorías en bioética}

En primer lugar, analizaremos el concepto de bioética y las corrientes que existen, para examinar después si la bioética debe ser considerada parte de la RSC.

El término bioética es un neologismo a partir del griego: bios-ethos (ética de la vida'), acuñado hace más de 40 años por el oncólogo Van Rensselaer Pot$\operatorname{ter}^{4}$ (Pérez Soba, 2008; Postigo, 2006; Sgreccia, 2007, entre otros). No obstante, aunque el nombre fuera acuñado por Potter, la bioética surgió en Estados Unidos no solo por su iniciativa:

«He elegido la raíz bio para representar el conocimiento biológico, la ciencia de los sistemas de los seres vivos; y ethics para representar el conocimiento del sistema de los valores humanos» (Potter). Potter detectó el peligro que corría la supervivencia de todo el ecosistema por la ruptura entre los dos ámbitos del saber: el científico y el saber humanístico.

(Sgreccia, 2007:5s)

Por otro lado, el título de este primer artículo, Bioethics. The science of survival (en castellano, Bioética, la ciencia de la supervivencia), es muy significativo, pues, como se verá posteriormente, la Responsabilidad Social Corporativa se presenta como una concepción de la empresa que permite el desarrollo sostenible, es decir, la «supervivencia» a nivel económico y social. Cabría ver aquí, en la raíz, en lo que serían sus metas, un primer nexo de unión entre bioética y RSC.

3 De aquellas empresas relacionadas especialmente con este campo. 4 Potter, V.R. (1970) «Bioethics. The science of survival» en Perspectives in Biology and Medicine 14, 127-153. 
Acudiendo propiamente a las definiciones de bioética, Sgreccia (2007: 24-25) aporta la definición de Reich, ${ }^{5}$ según la cual la bioética es:

el estudio sistemático de las dimensiones morales -incluido el enfoque moral, las decisiones, la conducta, los criterios, etc. - de las ciencias de la vida y de la salud, con el empleo de la variedad de metodologías éticas, en un planteamiento interdisciplinar.

En el Diccionario de bioética de Simón (2012: 126-135) encontramos que:

La bioética se ha definido como la parte de la ética que se refiere a las cuestiones planteadas por el desarrollo de las ciencias biomédicas en los ámbitos de la vida y de la salud ante problemas nuevos o antiguos modificándolos por las nuevas tecnologías...

La definición de bioética se basa en un modelo antropológico y ético que enmarca los principios generales y las orientaciones más concretas de una determinada perspectiva bioética. Siguiendo trabajos previos, los modelos de referencia que han dado lugar a las principales corrientes ( $y$ definiciones) bioéticas son el modelo sociobiologista, el modelo liberal-radical, el modelo pragmático-utilitarista, el modelo contractualista, la ética de los principios o principialismo y el modelo personalista (Sgreccia, 2007).

Esto muestra que, desde Potter, se plantea un problema de definición (Sgreccia, 2007:23-29), que parece seguir abierto. Aquí nos serviremos de las definiciones indicadas y partiremos de un modelo personalista para poder dar respuesta al objetivo de nuestro estudio, aunque por razones de espacio solo podemos remitir a la bibliografía para justificar nuestra elección. ${ }^{6}$

Tras indicar qué es la bioética y los modelos teóricos, definiremos la RSC para poder establecer la señalada relación entre bioética y RSC.

5 Como señala también Postigo (2006), «la primera edición de la Enciclopedia de Bioética se la definió como "el estudio sistemático de la conducta humana en el área de las ciencias de la vida y de la salud, examinadas a la luz de los valores y de los principios morales" W.T. Reich, (1978) Encyclopedia of Bioethics, Mac Millan, New York Reich, W.T.», pero como señala la autora esta definición fue cambiada por el mismo autor en posteriores ediciones, dada la controversia que suscitó.

6 Además de considerar el trabajo de Sgreccia (Manual de Bioética, 2007) como una buena síntesis a la que remitirse para establecer el marco teórico de nuestra investigación, cabe señalar que una adecuada valoración de las distintas definiciones de bioética implica un análisis del modelo que se tome como punto de partida. La discusión, en este sentido, queda abierta porque depende del modelo de referencia (antropológico) del que se parta. Pero no lo estaría en cuanto que objetivamente cada modelo justifica una determinada comprensión de la bioética (una definición). El trabajo de valoración de la adecuación de los modelos de referencia ha sido realizado, a nuestro juicio, de manera suficiente y definitiva por distintos autores, el primero de ellos, Elio Sgreccia, en el citado manual. La conclusión del estudio revela que solo la antropología personalista es un modelo adecuado y suficiente, puesto que se trata de una perspectiva holística en la consideración del ser humano, mientras que otras perspectivas adolecen de cierta unilateralidad en los planteamientos (únicamente la libertad, o la consideración de las consecuencias, o la dimensión social del ser humano, por ejemplo). 


\subsection{La Responsabilidad Social Corporativa (RSC)}

Para poder hablar de RSC debemos definir este concepto, sobre cuya comprensión encontramos distintas posturas. Así, Friedman (1970) señala que la verdadera responsabilidad de la empresa debe centrarse en «hacer tanto dinero como fuese posible para los accionistas», y salirse de ello socavaría los fundamentos de la sociedad. Sin embargo, la concepción actual de la RSC complementa, sin oponerse, la definición anterior. En el Libro Verde (Green Paper) de la Comisión Europea (2001:7) se afirma:

La mayoría de las definiciones de la responsabilidad social de las empresas entienden este concepto como la integración voluntaria, por parte de las empresas, de las preocupaciones sociales y medioambientales en sus operaciones comerciales y sus relaciones con sus interlocutores.

Ser socialmente responsable no significa solamente cumplir plenamente las obligaciones jurídicas, sino también ir más allá de su cumplimiento invirtiendo «más» en el capital humano, el entorno y las relaciones con los interlocutores...

La definición de la U.E. señala que las empresas deben recoger las preocupaciones sociales en sus operaciones comerciales y sus relaciones con sus interlocutores. Si se tiene esto en cuenta, se comprende que existe una preocupación social respecto a qué se investiga, con qué se investiga y, sobre todo, con quién se investiga. Si esto es así, como parece lógico, la bioética debería formar parte de la RSC de las empresas que guardan una relación directa con estas cuestiones. ${ }^{7}$

A continuación, se señalan algunos organismos divulgativos de la RSC.

\section{Pacto Mundial: Global Compact}

El Pacto Mundial es una iniciativa internacional propuesta por Naciones Unidas en el año 1999. Su objetivo es conseguir un compromiso voluntario de las entidades en responsabilidad social por medio de la implantación de diez principios basados en cuatro puntos: derechos humanos, normas laborales, medioambientales y de lucha contra la corrupción.

\section{AA1000AS}

El primer índice o norma AA1000 fue desarrollado en el 2003 por Accountability (Promoting Accountability for sustainable development), una organización internacional con sede en Londres. En concreto, AccountAbility desarrolla una

7 Este será uno de los argumentos utilizados para explicar el motivo de la investigación. El si debe existir dentro de la RSC un apartado sobre bioética, como reflejo de la respuesta de la RSC a una «preocupación social». 
serie de normas destinadas a medir y contrastar, entre otras cuestiones, la responsabilidad empresarial, de ahí la norma AA1000.

\section{SA8000: Social Accountabillity 8000}

Índice de la SAAS Social Accountability Accreditation Services Agencia, que nace en 1997 como parte de la Social Accountability International (SAI) norteamericana, y que ha ido adquiriendo más independencia con el correr de los años. Es una norma que valora las relaciones entre las empresas y los distintos agentes que se relacionan con ella: empleados, proveedores, o la mejora de las condiciones laborales de la empresa. Está basada en normas internacionales del trabajo, la Organización Internacional del Trabajo (OIT), la Declaración Universal de los Derechos Humanos y la Convención de las Naciones Unidas sobre los derechos del Niño.

\section{DJSI WORLD: Dow Jones Sustainability Indexes}

Nació en el año 1999 como resultado de la cooperación entre Dow Jones Indexes y SAM Group, para ofrecer a los agentes de la economía unos puntos de referencia fiables y objetivos, en cuanto al desempeño de las prácticas de RSC, por parte de las empresas líderes en sostenibilidad en el mundo. Actualmente, formar parte de este grupo exige, entre otros requisitos, la cumplimentación de un cuestionario exhaustivo que cubre la dimensión económica, pasando por las medidas de dirección y gestión de riesgos y crisis, hasta su código de conducta, políticas anticorrupción, la relación con los clientes, informes ambientales ecoeficientes, informes sociales, tanto en el ámbito de los empleados, como de la «ciudadanía corporativa», o aspectos filantrópicos.

Es relevante destacar que solo en este índice se ha encontrado la presencia del factor de la bioética, ${ }^{8}$ como factor dentro de la RSC, en concreto, para el caso de las empresas farmacéuticas.

\section{EFQM: European Foundation for Quality Management}

La Fundación Europea para la Gestión de Calidad (EFQM), según sus palabras: «Es una organización sin ánimo de lucro formada por organizaciones o empresas miembro y creada en 1988 por catorce importantes empresas europeas. Su Misión es ser la fuerza que impulsa la excelencia en las organizaciones europeas de manera sostenida».

8 Fuente: DJWI: <http://www.sustainability-indices.com/images/CSA_methodology_en_tcm1071338252.pdf $>$, consultado el 30 de junio del 2013 


\section{GRI: Global Reporting Initiative}

GRI es una iniciativa que busca la estandarización en la presentación de un Informe Global, en donde se incluyan aspectos tanto económicos y ambientales, como sociales. Es un estándar, reconocido y recomendado internacionalmente como índice, para potenciar y promover la RSC.

\section{FTSE4GOOD}

Como de Dow Jones Indexes surgió el DJSI, su competidor británico sacó el FTSE4Good, en línea con la RSC. FTSE es una compañía independiente de propiedad conjunta de Financial Times y la Bolsa de Valores de Londres (London Stock Exchange). Pretende ayudar a los inversores a identificar las empresas que cumplan con un estándar de responsabilidad corporativa, ambiental y social. Para ello, publica una serie de índices, entre los que se encuentra el FTSE4Good.

\section{Estado de la cuestión e hipótesis}

Como se ha señalado, la RSC ha sido abordada desde múltiples campos. No obstante, la relación con la bioética parece no ser uno de los más estudiados, a tenor de los pocos artículos encontrados que las relacionan directamente.

Entre ellos, cabe destacar, en primer lugar, el trabajo de Okada y Watanabe (2008), que esboza esta relación directa. Estos autores plantean la necesidad de introducir la bioética dentro de la RSE y denuncian que, si bien la Responsabilidad Social Corporativa es un tema de actualidad, pocos trabajos se han centrado en la situación específica de la RSC para cada industria. De entre estas industrias, destaca la biotecnológica, donde se investiga con genes y genomas, tanto de plantas y animales como de seres humanos, lo que suscita una preocupación en la sociedad acerca del modo de uso o aplicación, debido o indebido, en la investigación o el negocio que se deriva de estos materiales. Ante esto, los autores proponen un nuevo componente de la RSC para las empresas biotecnológicas: asumir la responsabilidad del uso de genes y genomas, y la biotecnología (GGB), a través de la adopción de una ética ambiental y una bioética. La ética ambiental se propone como la raíz de la responsabilidad ambiental que las empresas deben asumir. Los principios de bioética para la investigación genética deberán ser los tomados por la RSE para el uso de GGB, que llegan a proponer un modelo.

El trabajo presentado por Gutiérrez y Díaz (2013), además de enmarcar la bioética como parte de responsabilidad social en la gestión empresarial, propone incorporar la formación en bioética en todas las ramas de la ciencia. En esta línea se encuentra también el trabajo de López y Rivera-Cruz (2011), que señala la 
importancia de incluir principios bioéticos en la definición de lo que constituye una empresa, y estudia cómo influye esto en el desarrollo adecuado de la RSC.

En una relación no tan directa encontramos trabajos como el artículo de Smith (1988), «La biotecnología y la ley: ¿̇esponsabilidad social o libertad de investigación científica?» («Biotechnology and the law: social responsibility or freedom of scientific inquiry?»). El autor parte de una perspectiva sociobiologista, y señala la influencia que la teoría de la evolución darwiniana tiene en la genética: hace posible una práctica eugenésica que es valorada positivamente como herramienta para la mejora de la salud genética de los ciudadanos (o más exactamente, la selección de los ciudadanos en función de su patrón genético). Esta conclusión se apoya en una comprensión liberal de la actividad investigadora de estas empresas, pues señala como única restricción a la investigación científica lo que denomina medidas razonables.

Olivier et alii (2008) analizan el caso de EE. UU. en relación con la posible legislación respecto a medicina genómica personalizada. Estos autores acaban proponiendo diversas formas para que el desarrollo de los fármacos siga un proceso socialmente más responsable. Resumen estas estrategias en la promoción de un modelo de desarrollo de fármacos centrado en la sostenibilidad, que incorpora las necesidades financieras y sociales y presta atención a la compleja red de interacciones entre ambas. A su juicio, el modelo de mercado para el desarrollo de fármacos ya no es sostenible en su forma actual, proponen utilizar diversos métodos para asegurar la diversificación de mercados, la gestión de riesgos y la responsabilidad social. Se trata de un desarrollo de fármacos socialmente responsable que se ocupa de las desigualdades en la atención al paciente y la salud internacional.

Por otra parte, Griesse (2007) señala que el desarrollo de organismos modificados genéticamente (OGM) ha causado debate en todo el mundo y ha obligado a reevaluar las teorías de la responsabilidad social.

En relación con otros campos, está el artículo de Kuzma y Kuzhabekova (2011), en el se hace referencia a la relación entre RSC y nanotecnología. Los autores señalan cómo la creciente preocupación pública y la incertidumbre que rodea las tecnologías emergentes sugieren la necesidad de un comportamiento socialmente responsable de las empresas y el desarrollo e implementación de sistemas de vigilancia sobre ello. Los autores argumentan que la Responsabilidad Social Corporativa (RSC) es un aspecto importante de la supervisión de la nanotecnología, dada la falta de datos sobre los riesgos de los nanoproductos para el ambiente y la salud.

Mackie et alii (2006) describen cómo empresas biocientíficas han comenzado a poner en práctica mecanismos de RSC a favor de países en desarrollo, debido a 
que muchas empresas creen que tienen la responsabilidad moral de devolver a la sociedad, en lugar de centrarse solo en las ganancias. En dicho trabajo entrevistaron a más de 100 directivos de estas compañías para descubrir los problemas éticos a los que se enfrentan y qué mecanismos se están utilizando para tratar estas cuestiones. El trabajo presenta seis mecanismos de RSE que las empresas estudiadas están utilizando para abordar los problemas mundiales de salud en el mundo en desarrollo: el primero, los programas de donación de drogas; el segundo, las estrategias de precios; el tercero, el fortalecimiento de la capacidad local de salud; el cuarto, los sectores público y privado (APP); el quinto, la distribución de beneficios $y$, finalmente, el sexto, fundaciones benéficas.

Como se puede comprobar, aunque no todos manifiestan una relación directa entre RSC y bioética, esa preocupación sí subyace en la mayoría.

Proponemos dos hipótesis basándonos en estos artículos, especialmente en los de Kuzma y Kuzhabekova (2011), Mackie et alii (2006), Griesse (2007) y Olivier et alii (2008), en los que se muestra una preocupación pública por la aplicación de RSC por parte de las empresas que investigan con personas o con seres vivos con una relación directa con la bioética. Esta preocupación podría tener dos causas, que motivan las dos hipótesis de este trabajo: bien la falta de aplicación de RSC por este tipo de empresas, puesto que se propone su aplicación; o bien, que el hecho de que se apliquen medidas de RSC sensibilizaría a estas empresas hacia la necesidad de aplicar la bioética en sus comportamientos. Según lo anterior y el objetivo perseguido en esta investigación, las hipótesis que proponemos son las siguientes:

1. ${ }^{a}$ hipótesis: La adopción de medidas de la RSC por parte de las empresas favorece la aplicación bioética de estas.

2. ${ }^{a}$ hipótesis: Las empresas farmacéuticas y biotecnológicas son más tardías en la aplicación de políticas de RSC.

Para contrastar la primera hipótesis, se analiza la información publicada por las empresas farmacéuticas y biotecnológicas españolas cotizadas.

Para contrastar la segunda hipótesis, se hace un estudio empírico sobre las empresas cotizadas españolas, diferenciando las farmacéuticas y biotecnológicas, pues son las que se relacionan directamente con factores bioéticos. ${ }^{9}$ Se quiere contrastar si estas empresas, respecto del resto, adoptan en mayor o menor medida políticas de RSC.

En la medida en que estas hipótesis se comprueben, junto con los trabajos de investigación señalados, cabrá proponer la expansión de la aplicación de medidas de RSC en estas empresas, además de incluir un campo destinado a la bioética, para su mayor divulgación.

9 Estas coinciden con las analizadas para la primera hipótesis. 


\section{Muestra, metodología y modelo}

\section{Muestra}

La población que ha sido tomada como muestra son las empresas españolas que cotizan en bolsa, en concreto en el SIBE ${ }^{10}$ en el cierre del año 2007. La elección ha sido motivada por la búsqueda de disponibilidad en la información y de homogeneidad de los datos que se iban a tratar.

Para la recopilación de los datos necesarios (balances, cuenta de resultados, cotización, etc.), se ha utilizado la base Amadeus, Sabi, la Comisión Nacional del Mercado de Valores, las distintas bolsas españolas y el SIBE, entre otros.

De este modo, de las 184 empresas que cotizaban en bolsa en el SIBE a finales del 2007 (solo empresas españolas) y de las entidades financieras (bancos y cajas), por dispersión en los datos, queda una muestra de 122 empresas para analizar. El período considerado comprende los años del 2000 al 2009. ${ }^{11}$

En la Tabla 4.1 se presenta la muestra estudiada, clasificada por sectores. Dentro del sector de bienes de consumo, se encuentran 6 empresas clasificadas como «Productos farmacéuticos y biotecnología», que serán objeto de un análisis particular para la contrastación de la primera de las hipótesis, y cuyos datos son más recientes, pues la información ha sido obtenida en el 2013.

Tabla 4.1. Empresas de la muestra clasificadas según sector

\begin{tabular}{|l|c|c|}
\hline \multicolumn{1}{|c|}{ Sector $^{*}$} & $\begin{array}{c}\text { N. }{ }^{\circ} \text { total } \\
\text { de empresas }\end{array}$ & $\%$ \\
\hline 1. Petróleo y energía & 12 & $9,8 \%$ \\
\hline 2. Materiales básicos, industria y construcción & 29 & $23,8 \%$ \\
\hline 3. Bienes de consumo & 32 & $26,2 \%$ \\
\hline 4. Servicios de consumo & 21 & $17,2 \%$ \\
\hline 5. Servicios financieros e inmobiliarias & 23 & $18,9 \%$ \\
\hline 6. Tecnología y telecomunicaciones & 5 & $4,1 \%$ \\
\hline TOTAL & 122 & $100 \%$ \\
\hline
\end{tabular}

*Clasificación de las empresas por sector, según la presenta la Bolsa de Madrid.

Fuente: elaboración propia.

10 SIBE (Sistema de Interconexión Bursátil Español). Datos obtenidos del informe anual del 2007 sobre el IBEX presentado por Sociedad de Bolsas, S. A., empresa del Grupo BME, encargada de la gestión y funcionamiento del SIBE.

11 Estos datos servirán para la contrastación de la segunda hipótesis. 


\section{Metodología}

En este apartado se fija el modo de medir la RSC utilizando el índice de revelación o de divulgación propuesto por Chivite (2013).

En los apartados siguientes se presentarán, entre las empresas cotizadas, las clasificadas como «Productos farmacéuticos y biotecnología» dentro de las empresas de «Bienes de consumo», y en las que se analiza tanto la presencia de RSC, como de la bioética. Queda para estudios posteriores la ampliación del número de empresas analizadas que se relacionan con la bioética, como se señalaba en el apartado 3.1., «Bioética en la empresas», pero que no han sido consideradas aquí.

Finalmente, para contrastar la segunda de las hipótesis, se propone un modelo explicativo a través del planteamiento de una regresión lineal.

\section{Elíndice de revelación (índice de divulgación) para la medición de la RSC}

Se toma el índice propuesto por Chivite (2013) para la medición de la RSC por parte de las empresas cotizadas. Los índices que servirán de base para la creación del índice de RSC son el Pacto Mundial, las normas AA1000AS, SA8000, la inclusión en Dow Jones Sustainability Indexes (DJSI), el EFQM, el GRI (Global Reporting Initiative), el FTSE4Good y la mención y los informes de RSC, presentados por las empresas y hechos públicos.

Estos índices, ya explicados en el punto 2, representan variables que miden factores de RSC. Algunos de ellos se centran en características específicas relacionadas con la RSC y otros tienen un carácter más global, en todo caso, se trata de índices reconocidos que están directamente relacionados con la revelación por parte de las empresas de informes sobre RSC. En este apartado se presenta un indicador adicional, referido a la aparición de informes de RSC en las páginas web de las empresas, y el modo de calcular el indicador de RSC en este trabajo de investigación.

Respecto a la presentación en la web de la empresa de informes de RSC, se puede decir que, mientras que el resto de índices son «indicadores externos» a la propia empresa, dichos documentos pueden considerarse «indicadores internos», ya sean informes de RSC o de sostenibilidad.

Así, del agregado de los anteriores índices se obtendrá un índice único de RSC, para cada empresa y cada uno de los años del estudio.

Cada uno de los indicadores tomará valor 1 o 0 en función de si las empresas están incluidas o no, respectivamente. De modo que la variable agregada RSC 
total acumulativo será la suma de una serie de unos y ceros. Además, se tendrá también en consideración si en los años anteriores se han aplicado dichos índices.

A modo de ejemplo indicamos que, por ser 8 índices activos, el valor de la RSC el primer año considerado podría variar de 0 a 8 , mientras que el segundo año, de 0 a 16 (la suma de los 8 del primer año, más los 8 del segundo), y así sucesivamente. Esto no es del todo exacto, ya que el índice SA8000 solo muestra un valor (o solo suma una vez).

Esto se hace así porque se entiende que una empresa que lleva más tiempo aplicando políticas de RSC las tiene más asumidas que otra que empieza. Y, sobre todo, poder considerar los años con RSC es interesante por la propia naturaleza de la RSC que tiene un fuerte componente social. ${ }^{12}$

La expresión matemática, para el cálculo del índice de revelación, para cada empresa y cada año, es la siguiente:

$$
I_{a i}=\sum_{i=1}^{n} \sum_{j=1}^{m} I_{j i}
$$

Fuenteः Chivite (2013)

Donde $\mathrm{a}=$ la empresa, $\mathrm{i}=\mathrm{el}$ año considerado, $\mathrm{j}=\mathrm{el}$ índice de $\mathrm{RSC}, \mathrm{m}=\mathrm{el}$ total de índices, $\mathrm{n}=$ el total de años. A este índice de RSC se le llama RSC total acumulativo.

A modo de ejemplo, y para lograr una mejor comprensión, se señala el caso de Telefónica. En esta empresa se puede ver cómo su evolución ha sido continua y creciente. Comenzó en el 2002 con tres indicadores, en concreto con su adhesión al Pacto Mundial, su aparición en el DJSI y la publicación de un informe de RSC en su página web. Esto supone, según lo expuesto, que el índice RSC total acumulativo equivale a tres, puesto que no existen datos anteriores. El año siguiente, el 2003, vuelven a aparecer tres indicadores, en esta ocasión el RSC total acumulativo pasa a ser 6 , como resultado de la suma de los tres de este año más los tres anteriores. En el 2004, aparecen cuatro, puesto que es el acumulativo

12 Es decir, las prácticas de RSC no son un simple mecanismo más que incorporar dentro del sistema productivo de una empresa, como pudiera ser el cambio de software en el sistema de control de inventarios, sino que deben ser asumidas como algo importante por las personas que lo ponen en práctica. Así, para su adopción plena ha de ser asimilado, no solo aplicado con criterios puramente externos y numéricos, sino que ha de ser entendido como una nueva concepción de la propia empresa, que si bien es cierto que se manifiesta en hechos concretos, requiere de un tiempo de asimilación, puesto que, como se señalaba, es un cambio en la concepción de la propia esencia de la empresa misma, claro está, si se entiende y aplica bien. Por eso se considera importante, en este caso, que sea un índice acumulativo. 
de $10(3+3+4)$. Y así sucesivamente, llegando en el 2009 a aparecer en 7 de los 8 indicadores, con un RSC acumulativo de 38.

\section{Empresas del sector farmacéutico y biotecnológico}

A continuación, para contrastar la primera hipótesis, «la adopción por parte de las empresas de medidas de la RSC favorece la aplicación bioética por parte de las mismas», del sector clasificado como farmacéutico y biotecnológico, se pasará al análisis de la información publicada por estas empresas, buscando informes de RSC e información sobre bioética. ${ }^{13}$ En función de la información encontrada, podrá confirmarse o rechazarse dicha hipótesis.

Las empresas clasificadas como «Productos farmacéuticos y biotecnología», dentro de las empresas cotizadas españolas, ${ }^{14}$ son Grifols, S. A., Faes Farma, S. A. (FAES), Prim, S. A., Zeltia, S. A., Laboratorios Almirall, S. A., Laboratorios Farmacéuticos Rovi, S. A.

\section{Grifols $^{15}$}

Respecto de la RSC: presenta un apartado dedicado a ella y compuesto a su vez por seis subapartados: «Código de conducta», «Seguridad», «Salud», «Medio ambiente», «Fundació Víctor Grífols i Lucas», «Fundación Probitas», «Fundación José Antonio Grifols Lucas». Entre estos apartados se destacan dos‡ el de «Medio ambiente», donde se presenta la memoria de gestión ambiental, a través de informes anuales; y el dedicado, dentro de la propia RSC, a la Fundación Víctor Grífols que se dedica a la promoción de la bioética.

Respecto de la bioética: no solo habla de ella, sino que cuenta con la fundación mencionada, que promueve esta disciplina.

Comprobamos así cómo esta empresa aplica particularmente la Responsabilidad Corporativa adaptada a su propia actividad.

13 En este caso los datos se han obtenido en agosto del 2013. Particularmente la información ha sido obtenida de la documentación publicada en la página web de cada empresa. Se entiende que, por ser empresas cotizadas, con obligación de mantener informados a sus accionistas, mantienen actualizada la página web y vuelcan en ella lo que consideran «información relevante» en cumplimiento de Ley 26/2003, de 17 de julio.

Se considera información válida, y que el hecho de hacerla pública supone que es considerada relevante por la empresa. Si no se hace pública, por el contrario, no es relevante, y aunque se pudieran estar aplicando factores bioéticos por las mismas, al no ser publicados, se entiende que no son considerados relevantes y, por tanto, a efectos de esta investigación es como si no los aplicasen.

14 En concreto en el SIBE en el cierre del año 2007, como se ha señalado en el apartado anterior.

15 Toda esta información ha sido obtenida de la página web de Grifols en distintos apartados: <http://www. grifols.com >. 


\section{Faes Farma ${ }^{16}$}

Según la información publicada por la empresa:

Faes Farma es una compañía española con vocación internacional que investiga, produce y comercializa productos farmacéuticos y materias primas, exportando a más de 60 países...

No se ha encontrado apartados relacionados con la RSC ni con la bioética.

\section{$\operatorname{Prim}^{17}$}

Según la información publicada por la empresa:

Referencia en el mercado de suministros hospitalarios y ortopédicos, en fisioterapia, talasoterapia, termalismo, spa, rehabilitación, geriatría y ayudas técnicas. Se estructura para reforzar el desarrollo de sus valores culturales y empresariales: compromiso de calidad de servicio con el cliente, lealtad y transparencia con sus proveedores, y buen gobierno y responsabilidad social con los empleados y con el conjunto de la Sociedad.

Esta empresa, como en el caso de Faes Farma, no presenta apartados específicos para la RSC ni para la bioética. Lo único que señala en su propia definición es el hecho de buscar la responsabilidad social con sus empleados. Puesto que esto es lo único, no se considera que cuente explícitamente con RSC.

\section{Zeltia $^{18}$}

Respecto de la RSC: presenta un apartado dedicado a ella e informes anuales desde el 2009. En dicho apartado se señala:

Todos los ensayos clínicos se llevan a cabo de acuerdo con los principios éticos de la Declaración de Helsinki y los códigos de bioética nacionales e internacionales, tales como la Declaración de Oviedo, y en conformidad con las normas de Buena Práctica Clínica (GCP, Good Clinical Practice). Dichos ensayos son siempre evaluados y aprobados por los Comités Éticos de investigación clínica aplicables. Nuestras empresas procuran ser lo más respetuosas posibles con el medio ambiente...

16 Toda esta información ha sido obtenida de la página web de Faes Farma en distintos apartados: <http:// faes.es $>$. Consultado: 04/08/2013.

17 Información obtenida de en la página web: <http://www.prim.es/index.php/empresa.html $>$. Consultado: 04/08/2013.

18 Información obtenida de en la página web: <http://www.zeltia.es/quienes_somos.cfm>. Consultado: 04/08/2013 <http://www.zeltia.es/rsc.cfm>. 
Esta empresa, por tanto, hace mención a la aplicación de los códigos de bioética dentro de este apartado.

Respecto de la bioética: se ha encontrado dentro del apartado de la RSC, y no fuera de este.

\section{Almirall ${ }^{19}$}

Respecto de la RSC: presenta en un apartado dedicado a ella, pero denominado compromiso social, en el que diferencia sociedad, empresa y entorno (los tres componentes de la RSC adaptados).

Respecto de la bioética: dedica un apartado a la ética profesional, y en él se remite indirectamente a la bioética por su referencia a la Declaración de Helsinki, que propone una serie de principios éticos para la investigación médica y experimentación en seres humanos.

Almirall realiza ensayos clínicos de acuerdo a las normativas nacionales en los países donde son llevados a cabo, y siguiendo directrices y declaraciones internacionales, la Declaración de Helsinki y las Buenas Prácticas Clínicas...

\section{Rovi $^{20}$}

Respecto de la RSC: presenta un apartado en el que habla de RSC con la sociedad, con el medio ambiente, con los empleados, con los clientes, con los proveedores, con los accionistas. No obstante, no presenta informes anuales.

Respecto de la bioética: no dedica apartados específicos y tampoco parece hacer mención a la ética en la investigación.

A modo de resumen se presenta la Tabla 4.2, en la que se muestran las 6 empresas analizadas indicando si aplican o no RSC, especificando si presentan informes o solo se hace mención en su página web. Esto se considera importante, pues los informes anuales suponen una mayor implicación en este tipo de medidas, por el tiempo y recursos empleados que comporta. Por otro lado, se señala si consideran la bioética como factor para tener en consideración en el desarrollo de su actividad.

19 Información obtenida en la página web: <http://www.almirall.com>. Consultado: 04/08/2013.

20 Información obtenida de la página web: <http://www.rovi.es>. Consultado 05/08/2013. 
Tabla 4.2. Aplicación RSC y bioética por las empresas farmacéuticas-biotecnológicas

\begin{tabular}{|l|c|c|c|}
\hline \multicolumn{1}{|c|}{ Empresas } & RSC & Informes anuales & Bioética \\
\hline Grifols, S. A. & Sí & Informe & Sí \\
\hline Faes Farma, S. A. (FAES) & No & No & No \\
\hline Prim, S. A. & No & No & No \\
\hline Zeltia, S. A. & Sí & Informes & Sí \\
\hline Laboratorios Almirall, S. A. & Sí & Web & Sí $^{*}$ \\
\hline Laboratorios Farmacéuticos Rovi, S. A. & Sí & Web & No \\
\hline
\end{tabular}

* Se valora que considera factores bioéticos indirectamente, al mencionar la ética profesional y la Declaración de Helsinki.

Fuente: elaboración propia a partir de los datos extraídos de las empresas.

\section{Modelo explicativo propuesto}

Para analizar la segunda hipótesis planteada, si «las empresas farmacéuticas y biotecnológicas son más tardías en aplicar políticas de RSC», se aplica la siguiente regresión lineal:

RSC total acumulativo $=\beta o+\beta_{1}$ Industrias Bio $+\beta_{2} A \tilde{n} o R S C_{t-1}+\beta_{3} A_{i t}+$ $\beta_{4} R O A_{i t}+\beta_{5} I B E X_{i t}+\varepsilon_{t}$

Las variables que se analizan son:

RSC total acumulativo: variable dependiente que mide el nivel de Responsabilidad Social Corporativa, calculado según se ha explicado arriba. ${ }^{21}$

Industrias Bio: variable dummy, tomando valores 1 si la empresa es de este sector (farmacéuticas y biotecnológicas) y 0 en caso contrario. Esta variable permite contrastar la segunda hipótesis. Se espera signo negativo según lo propuesto, «las empresas farmacéuticas y biotecnológicas son más tardías en aplicar políticas de RSC», es decir, las empresas de este sector aplican menos RSC que el resto.

AñoRSC $\mathrm{t}_{\mathrm{t}-1}$ mide el número de empresas que adoptaron medidas de RSC el año anterior al estudiado, dentro de la muestra. Esta variable se establece para determinar si el hecho de que «otras» empresas apliquen RSC ha influido en la adopción por parte de las empresas de estas medidas. Se establece esta variable

21 En ocasiones por razones de espacio se ha denominado esta variable RSC total acumul. No se toman las otras variables dependientes propuestas por Chivite (2013), como eran las denominadas RSC total y Años con RSC. Entre las variables independientes solo se toman las más significativas como es la variable añoRSC denominada efecto contagio en dicho trabajo, el tamaño, Ibex y ROA. 
porque resultó fuertemente significativa en estudios previos Chivite (2013). El signo esperado es positivo, solo se toma el primer valor propuesto por dicho trabajo.

Ait: volumen del activo de la empresa i para el año $t$. Se considera que el tamaño de la empresa puede influir en la adopción de RSC, pues cuanto mayor es una empresa, normalmente, con más recursos cuenta para poner en práctica nuevos proyectos. El signo esperado para esta variable es positivo.

$R O A_{i t}:$ por otro lado, esta variable mide el cociente entre el beneficio neto, o después de intereses y el volumen de activo de la empresa $\left(\mathrm{BN} / \mathrm{A}_{\mathrm{it}}\right)$. El signo esperado es positivo.

$I B E X_{i t}$ : variable que toma el valor 1 o 0 en función de que la empresa i en el año t esté o no dentro del $\mathrm{IBEX}^{22}$. Se incluye esta variable porque se considera que las empresas del IBEX son un grupo significativo para el resto, cuyo comportamiento es más examinado por los distintos grupos de interés y, por tanto, con incentivos añadidos para aplicar las últimas tendencias del mercado. El signo esperado es positivo.

Incluimos estas últimas variables porque han sido constatadas como factores que influyen en la adopción de RSC de manera complementaria a través de múltiples trabajos, aunque no se hace un estudio particular de las mismas en este trabajo. ${ }^{23}$

\section{Resultados}

El primer objetivo consistía en evaluar si la adopción por parte de las empresas de medidas de RSC favorece o no la aplicación de principios bioéticos. Para ello, dividimos para el análisis las que no aplican RSC y las que sí, a fin de comprobar posteriormente si cada uno de estos grupos realiza consideraciones de tipo bioético.

De los datos mostrados en la Tabla 4.2, se puede observar que 4 de las 6 empresas (el 67\%) aplican de alguna manera RSC. ${ }^{24}$

Si se consideran en primer lugar las empresas que no aplican RSC, se observa que ninguna de ellas ofrece explícitamente consideraciones de carácter bioético. Es decir, el $0 \%$ hace referencia a la bioética. Sin embargo 3 de las 4 empresas que aplican RSC (el 75\%) sí la mencionan.

En cambio, aquellas que presentan informes de RSC y, por tanto, manifiestan un mayor compromiso con estas políticas, presentan una sensibilidad más

22 Datos obtenidos de los informes anuales sobre el IBEX presentados por Sociedad de Bolsas, S.A.

23 Se pueden encontrar ejemplos en el trabajo de Chivite (2013).

24 Cabe recordar que para este apartado, el análisis se hace para el 2013. 
o menos explícita hacia la bioética, e incluso una de ellas tiene una fundación dedicada al desarrollo y promoción de esta disciplina. En conclusión, el $100 \%$ de las empresas que publican informes de RSC tienen en consideración la bioética en el desarrollo de su actividad.

Según estos resultados, se acepta la hipótesis que sostiene una relación de dependencia entre la adopción por parte de las empresas de medidas de la RSC y la aplicación bioética por parte de las mismas.

Por otro lado, la regresión que se contrasta, para comprobar la segunda hipótesis, sobre si las empresas farmacéuticas y biotecnológicas son más tardías en aplicar políticas de RSC, es el modelo 4.2. Los datos de la muestra seleccionada se basan en 122 empresas y 10 años observables, lo que supone un total de 1.220 observaciones. Como no se dispone de todos los datos para todas las variables, se reduce la muestra al mínimo de datos disponible, y quedan así 1.093 observaciones completas. Se pasa a su contrastación utilizando el programa SPSS, del que se obtienen los siguientes resultados:

En la Tabla 5.1, los datos muestran que solo el 5\% de los datos corresponden a empresas de estos sectores ( 6 de 122). También destaca el valor del activo, ${ }^{25}$ como la cifra más elevada.

\section{Tabla 5.1. Estadísticos descriptivos}

\begin{tabular}{|c|c|c|c|c|c|c|c|}
\hline & & $\begin{array}{c}\text { RSC total } \\
\text { (acumul) }\end{array}$ & $\begin{array}{c}\text { Industrias } \\
\text { Bio }\end{array}$ & AñoRSC $_{t-1}$ & Activo Total & ROA & IBEX 35 \\
\hline \multirow[t]{2}{*}{$\mathrm{N}$} & Válidos & 1220 & 1220 & 1220 & 1093 & 1093 & 1220 \\
\hline & Perdidos & 0 & 0 & 0 & 127 & 127 & 0 \\
\hline \multicolumn{2}{|l|}{ Media } & 2,65 & ,05 & 31,70 & 4.602 .706 & ,0468 & ,21 \\
\hline \multicolumn{2}{|l|}{ Mediana } & ,00 & ,00 & 34,50 & 688.070 & ,0446 & ,00 \\
\hline \multicolumn{2}{|l|}{ Moda } & 0 & 0 & 1 & $366,(a)$ &,$- 894(a)$ & 0 \\
\hline \multicolumn{2}{|l|}{ Desv. típ. } & 5,770 & ,216 & 23,742 & 12.349 .552 & ,0948 & ,407 \\
\hline \multicolumn{2}{|l|}{ Varianza } & 33,291 & , 047 & 563,672 & $1,5251 \mathrm{E}+14$ & ,009 & , 165 \\
\hline \multicolumn{2}{|l|}{ Mínimo } & 0 & 0 & 0 & 366,00 &,- 894 & 0 \\
\hline \multicolumn{2}{|l|}{ Máximo } & 38 & 1 & 68 & 108.982 .000 & ,482 & 1 \\
\hline \multirow[t]{3}{*}{ Percentiles } & 25 & ,00 & ,00 & 1,00 & 207.635 & ,0176 & ,00 \\
\hline & 50 & ,00 & ,00 & 34,50 & 688.070 & ,0446 & ,00 \\
\hline & 75 & 2,00 & ,00 & 49,00 & 2.954 .168 & ,0788 & ,00 \\
\hline
\end{tabular}

Existen varias modas. Se mostrará el menor de los valores.

25 También es hizo el análisis para 1/activo total, y los datos no sufren variaciones muy significativas respecto a lo mostrado aquí. 
En la Tabla 5.2, se observan, como era de esperar, correlaciones entre el IBEX35 y el tamaño de la empresa. Por ello, se hace un análisis de sensibilidad, eliminando la variable de tamaño de la empresa, para contrastar si estos primeros resultados son válidos, y se trata de eliminar de ese modo el posible influjo de unas variables sobre otras.

Tabla 5.2. Correlaciones

\begin{tabular}{|c|c|c|c|c|c|c|c|}
\hline & & $\begin{array}{c}\text { RSC } \\
\text { total } \\
\text { (acumul) }\end{array}$ & $\begin{array}{c}\text { Industrias } \\
\text { Bio }\end{array}$ & AñoRSC $_{t-1}$ & $\begin{array}{c}\text { Activo } \\
\text { Total }\end{array}$ & ROA & IBEX 35 \\
\hline \multirow[t]{3}{*}{$\begin{array}{l}\text { RSC total } \\
\text { (acumul) }\end{array}$} & $\begin{array}{l}\text { Correlación de } \\
\text { Pearson }\end{array}$ & 1 &,$- 090\left({ }^{* *}\right)$ &, $416\left(^{* *}\right)$ &, $561(* *)$ & 022 &, $474\left({ }^{* *}\right)$ \\
\hline & Sig. (bilateral) & & ,002 &, 000 & ,000 & ,460 &, 000 \\
\hline & $\mathrm{N}$ & 1220 & 1220 & 1220 & 1093 & 1093 & 1220 \\
\hline \multirow[t]{3}{*}{ Industrias Bio } & $\begin{array}{l}\text { Correlación de } \\
\text { Pearson }\end{array}$ &,$- 090(* *)$ & 1 &, 000 &,$- 076\left(^{*}\right)$ & ,050 &,- 052 \\
\hline & Sig. (bilateral) & ,002 & & 1,000 & ,012 & ,098 & 071 \\
\hline & $\mathrm{N}$ & 1220 & 1220 & 1220 & 1093 & 1093 & 1220 \\
\hline \multirow[t]{3}{*}{ AñoRSCt-1 } & $\begin{array}{l}\text { Correlación de } \\
\text { Pearson }\end{array}$ &, $416\left(^{* *}\right)$ & ,000 & 1 &, $\left.098^{(* *}\right)$ &,$- 115(* *)$ & ,045 \\
\hline & Sig. (bilateral) &, 000 & 1,000 & & ,001 & ,000 & ,116 \\
\hline & $\mathrm{N}$ & 1220 & 1220 & 1220 & 1093 & 1093 & 1220 \\
\hline \multirow[t]{3}{*}{ Activo Total } & $\begin{array}{l}\text { Correlación de } \\
\text { Pearson }\end{array}$ &, $561\left(^{* *}\right)$ &,$- 076\left(^{*}\right)$ &, $098\left(^{* *}\right)$ & 1 & 021 &, $517(* *)$ \\
\hline & Sig. (bilateral) &, 000 & ,012 & ,001 & & ,479 &, 000 \\
\hline & $\mathrm{N}$ & 1093 & 1093 & 1093 & 1093 & 1093 & 1093 \\
\hline \multirow[t]{3}{*}{ ROA } & $\begin{array}{l}\text { Correlación de } \\
\text { Pearson }\end{array}$ & ,022 & ,050 &,$- 115(* *)$ & ,021 & 1 &, $108\left({ }^{* *}\right)$ \\
\hline & Sig. (bilateral) & ,460 & ,098 &, 000 & ,479 & &, 000 \\
\hline & $\mathrm{N}$ & 1093 & 1093 & 1093 & 1093 & 1093 & 1093 \\
\hline \multirow[t]{3}{*}{ IBEX 35} & $\begin{array}{l}\text { Correlación de } \\
\text { Pearson }\end{array}$ &, $474\left({ }^{* *}\right)$ &,- 052 & ,045 &, $\left.517^{* *}\right)$ &, $108\left(^{* *}\right)$ & 1 \\
\hline & Sig. (bilateral) &, 000 &, 071 & ,116 & ,000 &, 000 & \\
\hline & $\mathrm{N}$ & 1220 & 1220 & 1220 & 1093 & 1093 & 1220 \\
\hline
\end{tabular}

** La correlación es significativa al nivel 0,01 (bilateral).

* La correlación es significante al nivel 0,05 (bilateral).

Respecto de los resultados de la contrastación del modelo, los resultados se presentan en la Tabla 5.3. Estos presentan como significativas (sig. menor de $0,05)$ las siguientes variables: industrias Bio (con un $\beta$ negativo), empresa con RSC año anterior $\left(\right.$ AñoRSC $_{t-1}$ ), activo total e IBEX35; $y$, como no significativa, la variable $\mathrm{ROA}$. 
Tabla 5.3, Coeficientes modelo

\begin{tabular}{|c|c|c|c|}
\hline & $\begin{array}{l}\text { Coeficientes no } \\
\text { estandarizados }\end{array}$ & \multirow[b]{2}{*}{$t$} & \multirow[b]{2}{*}{ Sig. } \\
\hline & B & & \\
\hline (Constante) & $-1,985$ & $-8,203$ & 0,000 \\
\hline Industrias Bio & $-1,435$ & $-2,455$ & 0,014 \\
\hline AñoRSCt-1 & 0,095 & 17,221 & 0,000 \\
\hline Activo Total & 0,000 & 15,311 & 0,000 \\
\hline $\mathrm{ROA}$ & 2,017 & 1,495 & 0,135 \\
\hline IBEX 35 & 3,543 & 10,013 & 0,000 \\
\hline \multirow{5}{*}{$\begin{array}{l}\mathrm{N} \\
\mathrm{R}-\mathrm{sq}\left(\mathrm{R}^{2}\right) \\
\text { Ad,RSq } \\
\mathrm{F} \\
(\mathrm{P}-\mathrm{F})\end{array}$} & \multicolumn{3}{|c|}{1090} \\
\hline & \multicolumn{3}{|c|}{0,496} \\
\hline & \multicolumn{3}{|c|}{0,494} \\
\hline & \multicolumn{3}{|c|}{214,17} \\
\hline & \multicolumn{3}{|c|}{0,000} \\
\hline
\end{tabular}

A la luz de estos resultados, podemos aceptar la hipótesis planteada. La variable de industrias Bio resulta significativa y con un $\beta$ negativo, lo que implica que las empresas de estos sectores aplican menos RSC que el resto. Esto es así porque la variable de industrias Bio es una variable dummy que toma valor 1 en el caso de empresas farmacéuticas o biotecnológicas.

La capacidad explicativa del modelo, mostrada por la $\mathrm{R}^{2}$, es prácticamente del $50 \%$.

Por otro lado, dadas las correlaciones mostradas, se presenta el mismo análisis eliminando la variable de activo total, lo que permite eliminar el problema de la correlación entre dicha variable y la variable IBEX.

De su contrastación, se extraen los resultados presentados en la Tabla 5.4. Podemos comprobar que se mantienen unos resultados muy similares y se presentan como significativas (sig. menor de 0,05 ) las siguientes variables: industrias Bio (con un $\beta$ negativo), empresa con RSC año anterior $\left(\right.$ AñoRSC $_{t-1}$ ) e IBEX35; $y$, como no significativa, la variable ROA.

Se confirma, por tanto, la segunda hipótesis, de manera que las empresas farmacéuticas y biotecnológicas adoptan menos medidas de RSC que el resto, o bien, que son más tardías en su adopción.

La variable $R^{2}$ se reduce, puesto que desaparece una variable significativa, y la capacidad explicativa del modelo es de un $38 \%$. 
Tabla 5.4. Coeficientes modelo, sin activo

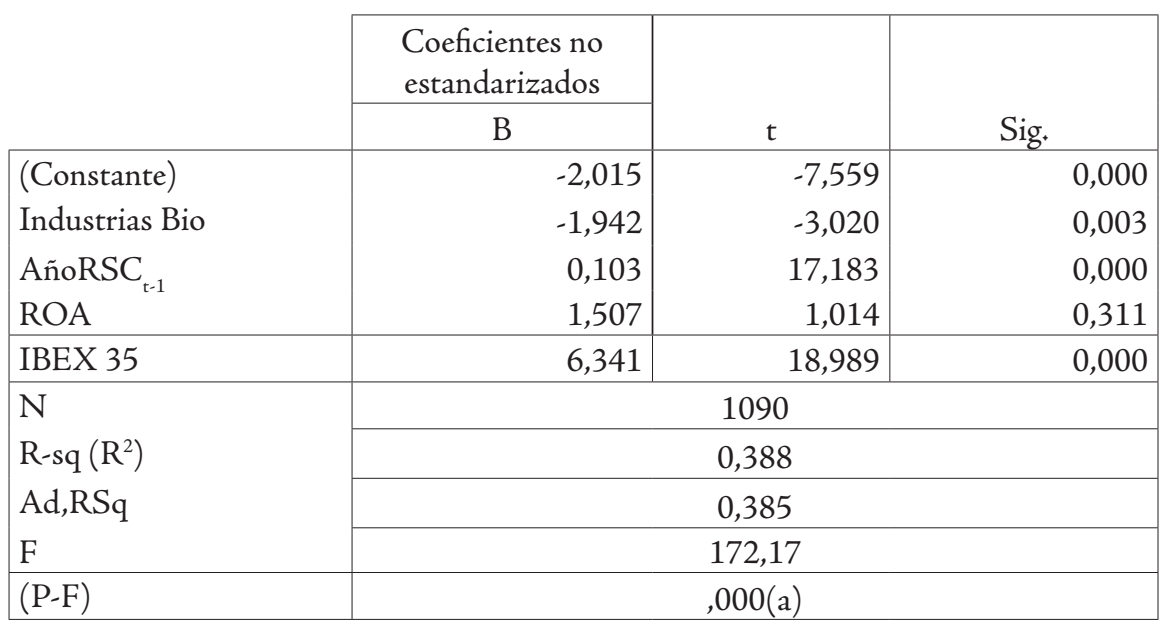

\section{Conclusiones}

El objetivo de este trabajo era fomentar la difusión y divulgación de la bioética, particularmente por su destacada importancia en las empresas que tienen una relación más directa con la investigación o promoción de la vida del ser humano.

Para ello, se propuso otra realidad paralela, que está cobrando en la actualidad gran relieve y una creciente adopción por parte de las empresas: la Responsabilidad Social Corporativa.

En este epígrafe final presentamos las conclusiones generales, la aplicación de los resultados a los que hemos llegado, las limitaciones y la dirección de futuras investigaciones.

En primer lugar, podemos afirmar que la RSC persigue ampliar los objetivos de la empresa más allá de la búsqueda de la rentabilidad económica, asumiendo su responsabilidad para con la sociedad con la que interactúa y de la que obtiene beneficios. Esta preocupación aparece en el marco teórico y en los índices que miden la RSC, desarrollados en el apartado dedicado al estado de la cuestión.

Existe hoy una verdadera preocupación social con respecto al campo médico y biotecnológico, y un deseo de evitar la manipulación o experimentación involuntaria con seres humanos. Puesto que la definición de RSC pide a las empresas integrar las «preocupaciones sociales», estas deberían incluir la bioética como parte de dicha responsabilidad, especialmente las empresas vinculadas con actividades de experimentación humana o biotecnológica. 
Con razón, el Dow Jones Sustainability Index (DJSI) incluía la bioética como factor dentro de la RSC para las empresas farmacéuticas, factor que, puntualmente, se ha empezado a integrar.

De ahí que se concluya que la bioética tiene cabida en la RSC, dando así una primera respuesta al objetivo marcado. ${ }^{26}$

Por otro lado, la inclusión de la bioética en la RSC, que experimenta una creciente adopción, ${ }^{27}$ revertirá en una expansión y divulgación de la misma bioética, como efecto colateral de la implantación de la RSC. Esto da también respuesta al objetivo de este trabajo.

Además, tras la aceptación de la primera hipótesis, se ha podido comprobar que la adopción por parte de las empresas de medidas de RSC favorece la aplicación bioética por parte de las mismas, y que la difusión de una ya está favoreciendo la aplicación o consideración de la otra. De hecho, las empresas que no aplicaban RSC no incluían bioética en su concepción, mientras que el $100 \%$ de aquellas que presentaban informes de RSC sí lo hacían.

No obstante, este tipo de empresas farmacéuticas-biotecnológicas parecen más remisas a adoptar RSC, de ahí que adquiera mayor importancia promover tales medidas entre estas empresas.

Llegados a este punto, se debe hacer mención de los distintos modelos bioéticos, pues no se trata solo de incluir la bioética dentro de la RSC, sino que su aplicación dependerá del modelo bioético que se asuma. No es lo mismo el modelo subjetivista o liberal-radical, el pragmático-utilitarista, la ética de los principios o el modelo personalista. De hecho, en el artículo mencionado de Smith (1988) se propone la aplicación de un modelo mixto (utilitarista y sociobiologista) a la empresa, pues se señala como única restricción a la investigación científica lo que denomina medidas razonables.

Por tanto, teniendo en cuenta que la inclusión de la bioética en la RSC supone la adopción de un modelo, se propone el modelo personalista como el más adecuado, siguiendo con la argumentación presentada por autores como Sgreccia (2007). De la adopción de otro tipo de modelos se derivarían muchos problemas, señalados por Sgreccia (2007) ${ }^{28}$

Una vez expuestas las conclusiones, presentamos la aplicación de los resultados alcanzados. En primer lugar, se denuncia la falta de aplicación de la bioética en empresas directamente relacionadas con este ámbito (en el sector farmacéu-

26 Cabría aquí mayor y mejor argumentación, pero se deja para trabajos posteriores.

27 Se pueden consultar los propios indicadores GRI, Pacto Mundial, DJSI, etc., o trabajos como Chivite (2013) o Server y Capó (2009), entre otros.

$28 \mathrm{La}$ argumentación en este sentido es una de las posibles líneas de investigación que abre este trabajo. 
tico-biotecnológico, objeto del análisis), que deberían incorporarla al desarrollo de su actividad.

Del mismo modo se ha intentado mostrar la relación directa entre RSC y la aplicación de la bioética, dentro de las empresas objeto de estudio, de manera que se propone aprovechar la inercia de una para fomentar la otra, valorando esto como una de las aportaciones de este trabajo.

Por lo anterior, se aconseja incluir en el apartado social de la RSC un epígrafe destinado a la bioética.

Si bien es aconsejable un criterio común de RSC para las empresas en general, se propone un apartado específico para la bioética para los sectores especialmente relacionados con esta. Puesto que solo uno de los índices estudiados, el DJSI, incluía este apartado, se observa que no se trata de una práctica extendida.

El hecho de que se plantee incluir este factor dentro del apartado «Social», está en línea con lo expuesto, porque los temas bioéticos son una preocupación social y, por otro lado, porque no solo el DJSI al incluir la bioética la enmarca dentro de la dimensión social, sino que el resto de índices siguen el mismo camino, por ejemplo, el Pacto Mundial (ver los principios 1 y 2, bloque de derechos humanos).

En segundo lugar, se propone incentivar la adopción de medidas RSC por parte de las empresas que tienen una relación más directa con la investigación o promoción de la vida del ser humano.

Como se ha expuesto, la RSC contribuye a la aplicación de la bioética y, si el objetivo de esta investigación es su difusión, se propone, por tanto, que se tomen medidas en esta línea.

A fin de ofrecer una perspectiva de continuidad, quisiéramos señalar, para terminar, las limitaciones de este estudio, que es solo el comienzo de un camino de investigación, y la orientación de posibles investigaciones futuras. Es necesario un estudio más profundo de los campos tratados, tanto en la fundamentación como en la extensión de las empresas analizadas, de las variables consideradas, etc. Principalmente se destaca la necesidad de profundizar en la argumentación que defienda una adopción del modelo personalista como aplicación bioética, dentro de la RSC.

Por otro lado, queda pendiente el desarrollo de un modelo concreto, respecto de la aplicación y valoración de la bioética, dentro del apartado social de la RSC, como se ha propuesto.

Por todo lo dicho, este trabajo es una puerta de entrada a un campo de investigación poco transitado. De ahí que este artículo, en deuda con trabajos precedentes, quiera ser una contribución a estudios posteriores y una ayuda para promover la bioética dentro del mundo de la empresa. 


\section{Bibliografía}

Alberich, T. (2014). «Movimientos sociales, responsabilidad corporativa e inclusión social en la Globalización», Revista Internacional de Sociología (RIS), 72 (1): 113-132. DOI:10.3989.

Altisent, R. y Martín, M.N. (2001). «Comités de ética en atención primaria», Atención Primaria, 8: 550-553.

Carroll, A.B. (1999). "Corporate Social Responsibility: Evolution of a Definitional Construct», Business \& Society, 38 (3): 268-295.

Chivite, M.P.; García, B.; Tua, J. y Enciso, V. (dir.) (2013). Determinantes y consecuencias económicas de la transparencia financiera: el caso particular de la Responsabilidad Social Corporativa, Universidad Católica de Ávila, Facultad de Ciencias Sociales y Jurídicas.

Comisión Europea (2001). «Green paper-Promoting a European framework for corporate social responsibility», Diario oficial de la Unión Europea (127).

Friedman, M. (1970). «The Social Responsibility of Business in to Increase its Profits», The New York Times Magazine.

Griesse, M. A. (2007). «Developing social responsibility: Biotechnology and the case of DuPont in Brazil», Journal of business ethics, 73 (1): 103-118.

Gutierrez, Huby, A. M., y de Díaz, E. V. R. (2013). «Bioética y Responsabilidad Social Corporativa, consideración para la formación integral universitaria», Revista de la Facultad de Ciencias Contables, 21: 19-27.

Kuzma, J. \& de Kuzhabekova, A. (2011). «Corporate social responsibility for nanotechnology oversight», Medicine, Health Care and Pbilosophy, 14: 407419.

Ley 14/2007, de 3 de julio, de Investigación Biomédica. BOE n. ${ }^{\circ}$ 159, de 4 de julio de 2007. 28826-48.

López Paláu, S. y de Rivera-Cruz, B. (2011). «El razonamiento bioético y la orientación hacia responsabilidad social empresarial de los estudiantes de negocios», Forum Empresarial, 16 (1): 59-102.

Mackie,J. E.; Taylor, A. D.; DaAr, A.S. \& De Singer, P. A. (2006). «Corporate social responsibility strategies aimed at the developing world: perspectives from bioscience companies in the industrialised world», International journal of biotechnology, 8 (1): 103-118.

McGuire, J.B.; Sundgren, A. \& Schneeweis, T. (1988). «Corporate Social Responsibility and Firm Financial Performance», Academy of Management Journal, 31 (4): 854-872. 
McWilliams, A.; Siegel, D.S. \& Wright, P.M. (2006). «Corporate Social Responsibility: Strategic Implications», Journal of Management Studies, 43 (1): 1-18.

Okada, Y. \& de Watanabe, K.N (2008). «Social responsibility for the use of genes, genomes and biotechnology in biotechnology companies: A commentary from the bioethical viewpoint», Journal of Commercial Biotechnology, 14 (2): 149-167.

Olivier, C.; Williams-Jones, B.; Godard, B.; Mikalson, B., \& de Ozdemir, V. (2008). «Personalized medicine, bioethics and social responsibilities: Rethinking the pharmaceutical industry to remedy inequities in patient care and international health», Current Pharmacogenomics and Personalized Medicine (Formerly Current Pharmacogenomics), 6 (2): 108-120.

Pérez-Soba, J.J. (2008). «Bioética de los principios», Cuadernos de Bioética, XIX: 43-55.

Porter, M.E. \& Kramer, M.R. (2006). «Strategy \& Society: The Link Between Competitive Advantage and Corporate Social Responsibility», Harvard business review, 84 (12): 78-92.

Postigo, E. (2006). «Concepto de Bioética y corrientes actuales», Bioética en la Red. Disponible en: <http://www.bioeticaweb.com>.

Potter, V.R. (1970). «Bioethics. The science of survival», Perspectives in Biology and Medicine, 14: 127-153.

Real Academia de Ciencias Económicas y Financieras (RACEF) (2007). La responsabilidad social de la empresa (RSE): Propuesta para una nueva economía de la empresa responsable y sostenible, Real Academia de Ciencias Económicas y Financieras, Barcelona.

Server, R. J. y Capó, J. (2009). «La Responsabilidad Social Empresarial en un contexto de crisis. Repercusión en las Sociedades Cooperativas», CIRIECEspaña, Revista de Economía Pública, Social y Cooperativa, 65:7-31.

Sgreccia, E. (2007). Manual de Bioética. I Fundamentos y ética biomédica, Madrid, Biblioteca de Autores Cristianos.

Simón Váquez, C. (2012). Nuevo diccionario de Bioética, Burgos, Editorial Monte Carmelo.

Smith, G. (1988). «Biotechnology and the lawः social responsibility or freedom of scientific inquiry?», Mercer law review, 39 (2): 437-460. 


\section{Páginas web consultadas}

AccountAbility. Disponible en: <http://www.accountability.org/>

Asociación Española del Pacto Mundial 2008, ASEPAN - El Pacto Mundial. Disponible en: <http://www.pactomundial.org>

Comisión Nacional del Mercado de Valores (CNMV), Informes entidades cotizadas. Disponible en: <http://www.cnmv.es/portal/Consultas/BusquedaPorEntidad.aspx > $\quad<$ http://www.cnmv.es/portal/Publicaciones/CodigosGovCorp.aspx>

Corporate Register, Informes. Disponible en: <http://www.corporateregister. $\mathrm{com} />(2007-2011)$

Dow Jones Sustainability Indexes (DJSI). Disponible en $\div$ http //www.sustainability-indices.com/>

European Foundation for Quality Management (EFQM), Informes. Disponible en: <http://www.efqm.org >. El partner en España. Disponible en: http://<www.clubexcelencia.org >

FTSE The Index Company* (FTSE4Good), Informes. Disponible en: <http:// www.ftse.com/>; <http://www.ftse.com/Indices/FTSE4Good_Index_Series/index.jsp>

Global Reporting Initiative (GRI), Informes. Disponible en: < https://www.globalreporting-org/Pages/resource-library+aspx?resSearchMode=resSearchM odeText\&resLangText $=$ Spanish\&resPageNumber $=1>(2012)$

International Organization for Standardization (ISO), ISO 26000 - Social Responsibility. Disponible en: <http://www.iso.org/iso/home/standards/ management-standards/iso26000.htm $>$ (2011)

Laboratorios Almirall S. A., Página e información publicada por la compañía. Disponible en: <http://www.almirall.com/webcorp2/cda/index.jsp>

Laboratorios Farmaceuticos Rovi S. A., Página e información publicada por la compañía. Disponible en* <http //www.rovi.es/>

FAES Farma S. A., Página e información publicada por la compañía. Disponible en: <http $/ /$ faes.es/>

Grifols S. A., Página e información publicada por la compañía. Disponible en: $<$ http://www.grifols.com/es/web/international/home>

Prim S. A., Página e información publicada por la compañía. Disponible en: $<$ http://www.prim.es/>

Social Accountability International (SAI) 04/03/2011-last update, The SA8000 Standard. Disponible en: <http://www.sa-intl.org/index. $\mathrm{cfm}$ ? fuseaction $=$ Page $\cdot$ view $\mathrm{Page} \&$ page $\mathrm{Id}=937 \&$ parent $\mathrm{ID}=479 \&$ node $\mathrm{ID}=1>[2011$, marzo $]$. 
Sociedad de Bolsas. Sistema de Interconexión Bursátil (SIBE), Informes anuales. Disponible en: <http://www.sbolsas.es/>

ZELTIA S. A., Página e información publicada por la compañía. Disponible en: $<$ http://www.zeltia.es/> 